\title{
The General Surgery Residency Program in Italy: a changing scenario
}

\author{
Marco Montorsi ${ }^{1} \cdot$ Nicolò De Manzini ${ }^{2}$
}

Received: 24 June 2019 / Accepted: 26 June 2019 / Published online: 6 July 2019

(C) Italian Society of Surgery (SIC) 2019

Disaffection in general surgery specialty has steadily increased over the last years due to multiple factors:

- general perception of a long and difficult career with complete surgical autonomy reached only after several years of practice;

- increasing medico-legal problems due to a more and more conflictual patient-doctor relationship;

- lower quality of life as compared to other medical and surgical specialties;

- career progression sometimes affected by non-technical factors (political reasons);

- increasing workload due to personnel shortage.

All these reasons had a great influence even on the choice of the postgraduate residency in General Surgery when doctors enroll for the national examination.

In fact, the total amount of available places lightly decreased in the last years with doctors choosing the places ranking below in the general list of those who passed the examination.

This is particularly humbling as in the same period surgery had a terrific increase in technological advances and scientific knowledge opening exciting and new scenarios for doctors willing to be surgeons.

The surgical education pathway is therefore the most important factor if we want to reverse this situation and assist a renaissance of surgery in our country.

A surgical specialty after graduation should imply that:

- the resident is motivated to tackle a complex and varied path;

- the medical training should also ensure the creation of a "finished product"; in other words, a surgeon ready to

\footnotetext{
Marco Montorsi

marco.montorsi@humanitas.it

Humanitas University, Milan, Italy

2 University of Trieste, Trieste, Italy
}

be "placed on the market", armed with cultural competences, decision-making skills and practical autonomy.

Of course, this does not mean that a young surgeon should be able to face autonomously a very complex surgery, but it is no longer tolerable that newly trained specialists do not achieve adequate technical skills in their curriculum, a curriculum that is also validated by the Ministry of Education, University and Research.

In the past, in our country, the residency program was unpaid, with the resident not working in the units where the training was based; teaching was almost only theoretical and the practice was devolved to the goodwill of the chief of the assigned unit, with neither quality or quantity control.

When compared to other European countries, a serious training "gap" emerged, especially concerning surgical activity, a prerogative of few. Undoubtedly, the anomalous organization in our university system provided units which, in some cases, could only offer a limited caseload, so contributing to many of these discrepancies.

Thus, the reform of the residency training [1] tried to reorganize our educational system on the basis of what is already active in other European nations.

First, the reform set out a regulation of the conditions of the residency programs.

Second, a contractual relationship was established with the residents, implying obligations, rights and a salary.

Third, for each medical area (medicine, surgery, services) and for each residency program, the theoretical and practical training goals were defined, specifying the theoretical knowledge and the minimum number and type of practical procedures to be included in the residency curriculum.

Fourth, the percentage of procedures carried out by a resident was established according to the unit's surgical potential to avoid numerically unsustainable conditions. As a corollary to this point, the rules for setting up a training network have since been laid out and defined as a ensemble of university hospital units linked to public and private hospitals to provide a global range of operations sufficiently varied and numerous to allow at least $20 \%$ of the surgical procedures to be carried out by the residents. 
Today, a resident in General Surgery in Italy must acquire specific knowledge in surgical anatomy, pathophysiology, perioperative treatment and he or she must attend a common trunk of general, plastic and pediatric surgery. Above all, a resident needs to participate in at least 425 surgical operations, classified as high, medium and low complexity, as first operator, respectively, in roughly one-third of the cases. The classification of the interventions by complexity has been defined by the National College of Professors of Surgery and should be applied in individual schools. In addition, surgical and other activities related to the surgical curriculum must be reported in an appropriate register, preferably computerized and validated by the School Director. It is also advisable for a resident to spend a period of his or her training abroad, with an active role in centers of recognized value. The National Observatory of Residency Schools requires regional observatories to monitor the correct application of the law in Italian residency programs.

Considering this brief summary of the law and its related application decrees which reform residency training, we must now ask ourselves if the law is being fully applied at a national level, taking into considerations the stringent observations made by Physicians organizations [2,3], individual young colleagues, and the census of the Schools of General Surgery Board.

To date, certain core criticisms have arisen, concerning the following issues:

- the main Residency program only provides General Surgery, Pediatric Surgery and Plastic Surgery, without taking into account other surgical specialties close to General Surgery such as Urology, Orthopedics, Gynecology, Vascular and Thoracic Surgery;

- rotations in these units are not foreseen by the law and are organized only thanks to the goodwill of the Specialty Directors;

- a poor management of the theoretical part of teaching, which should officially give room not only to upfront lectures but also to other didactic activities such as seminars, multidisciplinary events, literature reviews and journal clubs;

- an inadequate control of the resident's surgical activity, frequently out of compliance with the minimum level expected in the Ministerial Decree.

Therefore, to fully meet the criteria established by the European Union of Specialist Doctors (UEMS), the Italian Observatory will quickly need to report on inadequate institutions and improve the existing critical areas.

The situation concerning residency recruitment has further deteriorated due to a new admission system to residency programs, where candidates' pre-degree experience or their specific aptitude as a future trainee is not taken into account [4].

Moreover, medical graduates are now free to select any of the available specialties by simply ordering their preferences. And finally, a resident enrolled in a given surgical school and dissatisfied with its teaching program may retake the national admission test and, if successfully passed, leave the first specialty school, causing the school to loose its allocated ministerial funding.

How can we at best resolve these inadequacies? In our opinion, admission should require:

- a preselection evaluation based on the motivation of the future trainee, also considering specific previous experiences;

- the candidate should be allowed to only choose a main branch of medicine, i.e., medical, surgical, services. Consequently, this would require the resident to follow a common trunk training, closer to the needs of the real world, prior to choosing to enter a specific surgical specialty.

We do hope that these considerations could be helpful in increasing the level of surgical training in our country together with a cogent application of the rules of the new law of reform of postgraduate education [1].

In conclusion, we strongly believe that a strict control and rigorous assessment of the entire surgical training programs as clearly indicated by the new law of reform of the postgraduate education is of utmost importance. A newly structured cooperation between academic and non-academic professionals with clear and well-depicted roles and duties may be the most important advancement to accomplish this goal.

\section{Compliance with ethical standards}

Conflict of interest The author(s) declare that they have no competing interests.

Research involving human participants and/or animals This manuscript does not involve research on human subjects or animals.

Informed consent For this type of study formal consent is not required.

\section{References}

1. D.I. 13 giugno 2017, G.U. n. 163 del 14 luglio 2017

2. Indagine sulla formazione specialistica, Associazione Italiana Giovani Medici (SIGM)

3. M. Minerva, Associazione Liberi Specializzandi, personal communication

4. Decreto 10 agosto 2017, n. 130, G.U. n. 208 del 6 settembre 2017

Publisher's Note Springer Nature remains neutral with regard to jurisdictional claims in published maps and institutional affiliations. 